\title{
Role of 8-nitro-cGMP and its redox regulation in cardiovascular electrophilic signaling
}

\author{
Motohiro Nishida $^{1,2,3}$, Takashi Toyama ${ }^{1,3}$, and Takaaki Akaike ${ }^{4}$
}

${ }^{1}$ Division of Cardiocirculatory Signaling, Okazaki Institute for Integrative Bioscience (National Institute for Physiological Sciences), National Institutes of Natural Sciences, Aichi 444-8787, Japan, ${ }^{2}$ PRESTO, Japan Science and Technology Agency (JST), Kawaguchi, Saitama 332-0012, Japan, ${ }^{3}$ Department of Drug Discovery and Evolution, Graduate School of Pharmaceutical Sciences, Kyushu University, Fukuoka 812-8582, Japan, ${ }^{4}$ Department of Environmental Health Sciences and Molecular Toxicology, Tohoku University Graduate School of Medicine, Sendai 980-8575, Japan.

Corresponding author:

Motohiro Nishida, Ph.D.

Division of Cardiocirculatory Signaling, Okazaki Institute for Integrative Bioscience (National Institute for Physiological Sciences), National Institutes of Natural Sciences, 5-1 Higashiyama, Myodaijicho, Okazaki, Aichi 444-8787, Japan.

Telephone and fax: +81-564-59-5560

E-mail: nishida@nips.ac.jp

Content: 7,338 words

Abstract: 150 words

Figures: 4 


\begin{abstract}
Structural and morphological changes of the cardiovascular systems (cardiovascular remodeling) are a major clinical outcome of cardiovascular diseases. Many lines of evidences have implied that transfiguration of reduction/oxidation (redox) homeostasis due to excess production of reactive oxygen species (ROS) and/or ROS-derived electrophilic metabolites (electrophiles) is the main cause of cardiovascular remodeling. Gasotransmitters, such as nitric oxide (NO) and endogenous electrophiles, are considered major bioactive species and have been extensively studied in the context of physiological and pathological cardiovascular events. We have recently found that hydrogen sulfide-related reactive species function as potent nucleophiles to eliminate electrophilic modification of signaling proteins induced by NO-derived electrophilic byproducts (e.g., 8-nitroguanosine 3',5'-cyclic monophosphate and nitro-oleic acid). In this review, we discuss the current understanding of redox control of cardiovascular pathophysiology by electrophiles and nucleophiles. We propose that modulation of electrophile-mediated post-translational modification of protein cysteine thiols may be a new therapeutic strategy of cardiovascular diseases.
\end{abstract}

Keywords: reactive oxygen species, electrophile, nucleophile, $G$ protein, cardiovascular remodeling 


\section{Contents}

1. Introduction: Redox homeostasis in the cardiovascular system

1.1.Role of electrophiles and nucleophiles in redox signaling

1.2.Regulation of ROS signaling in the cardiovascular system

1.3.Formation, function, and metabolism of 8-nitro-cGMP

1.4.Redox control of vascular tone

2. Redox control of $\mathrm{G}$ protein signaling

2.1. G proteins and cardiovascular remodeling

2.2. Redox control of $G$ protein cycling

2.3. Regulation of $\mathrm{G}$ protein activity by PTMs

3. Redox control of cardiac remodeling

3.1. Formation of 8-nitro-cGMP in cardiac cells

3.2. Cardiac cell senescence via S-guanylation of H-Ras

3.3. Prevention of cardiac senescence via elimination of electrophiles by reactive sulfur species

4. Conclusions 


\section{Introduction: Redox homeostasis in the cardiovascular system}

\subsection{Role of electrophiles and nucleophiles in redox signaling}

Redox homeostasis, a redox-based cellular metabolism, is maintained in a precise balance between reactive oxygen species (ROS) and antioxidant systems, counteracting each other during diverse cellular metabolism and signaling [1]. Physical and chemical stimuli activate ROS-dependent signaling pathways via several ROS-producing enzymes. There are two major sources of these enzymes, NADPH oxidase (Nox) and the mitochondrial electron transport chain. Additionally, xanthine oxidase, aldehyde oxidase, cytochrome P450, urate oxidase, D-amino oxidases, and uncoupled NO synthases (NOSs) are involved [2-4]. Each enzyme forms an original protein signaling complex with specific sensor/effector proteins to activate specific signaling pathways. A decline in ROS signaling is mainly regulated by ROS-scavenging enzymes (e.g., superoxide dismutase [SOD], catalase, glutathione peroxidase [GPx], and peroxiredoxins) [5-7]. SOD is an enzyme that reacts with superoxide, and catalase and peroxiredoxins catabolize hydrogen peroxide $\left(\mathrm{H}_{2} \mathrm{O}_{2}\right)$. GPx is a selenium-dependent enzyme that contains a selenium atom incorporated within the selenocysteine residue [7]. GPx scavenges $\mathrm{H}_{2} \mathrm{O}_{2}$ in the presence of reduced glutathione (GSH). Because the intracellular concentration of GPx-coupled GSH is $1-3 \mathrm{mM}$ and patients with selenium deficiency have heart failure, most $\mathrm{H}_{2} \mathrm{O}_{2}$ in the cardiac system is thought to be eliminated through GPx [8]. Additionally, the thioredoxin (Trx) and glutaredoxin family of proteins play an essential role in maintaining redox homeostasis by NADPH-dependent reduction of disulfide bridges in various target proteins. Deletion of every ROS-scavenging enzyme results in several cardiovascular abnormalities or a shorter life span [9-12]. Therefore, maintenance of redox homeostasis is predominantly regulated not only by a formation of ROS via ROS-generating enzymes, but also by a complex web of antioxidant molecules.

In addition to ROS-producing/scavenging systems, the reversibility of redox-dependent post-translational modification (PTM) of proteins regulates a spatiotemporal diversity of ROS signaling in cells [13]. The ROS signaling system, as an unstable primary signal of ROS, transforms to a stabler secondary signal. During this process, intracellular nucleic acids, nucleotides, lipids, and cysteine (Cys) sulfhydryl-containing proteins function as chemical sensors to interact with ROS/NO. The secondary signal molecules formed via reaction of ROS/NO and intracellular sensor molecules are termed "electrophiles”. Electrophiles also target Cys sulfhydryls to form stable covalent modification of proteins, leading to continuous activation of redox-dependent signaling. We recently found that hydrogen sulfide-related reactive 
sulfur molecules function as a potent nucleophile to quench electrophilic PTM of proteins [14]. The physiological functions of hydrogen sulfide-related reactive sulfur molecules have been well documented in the vascular system [15-17]. Balancing electrophile and nucleophile signaling may provide a new aspect of redox control of cardiovascular homeostasis.

\subsection{Regulation of ROS signaling in the cardiovascular system}

Cardiovascular diseases are the leading cause of morbidity and mortality in developed countries. Many lines of evidence have suggested that ROS mediate the development of cardiovascular remodeling induced by neurohumoral factors and hemodynamic load via oxidative PTM of intracellular signaling proteins $[18,19]$. The above-mentioned ROS-generating enzymes are found to mediate agonist-induced activation of signaling proteins via oxidation of sulfur-containing amino acids (methionine and Cys), which are found at active or allosteric sites of effector proteins $[1,20]$. Several studies using gene-targeting mice have shown that ROS and/or reactive nitrogen oxides (RNS) function as a cytotoxic factor, and also act as an essential signaling mediator for maintaining physiological functions. This concept is supported by the surprising discovery that its counterpart, reductive stress, which is caused by an increase in reduced $\mathrm{GSH}$, contributes to cardiomyopathy triggered by protein aggregation [20,21]. Additionally, our recent study showed that irreversible Cys modification by electrophilic byproducts, such as nitrated cyclic nucleotide (e.g., 8-nitroguanosine 3',5'-cyclic monophosphate [8-nitro-cGMP]), generated via reactions of ROS, RNS, and intracellular guanine nucleotide, acts as an important mediator of chronic heart failure [14]. Therefore, reversibility of protein modification by ROS or ROS-derived electrophiles may determine the transition from adaptation to maladaptation of the heart against oxidative stress. These findings are of great value because they provide a new aspect for the management of cardiovascular diseases.

The spatiotemporal property of ROS signaling functions depends on the ROS production system and chemical reactivity, which defines the molecular reaction environment. To elucidate the mechanisms of ROS signal production and sensing, the structure and function of ROS-producing enzymes need to be analyzed. Among ROS-producing enzymes, the structure and function of Nox family proteins have been well studied [22]. The activation of Nox2 primarily depends on intracellular $\mathrm{Ca}^{2+}$ concentrations, where an increase in $\mathrm{Ca}^{2+}$ activates a small GTP-binding protein, Rac, and protein kinase C (PKC)-mediated phosphorylation of $\mathrm{p} 47^{\text {phox }}[22,23]$. The cytosolic regulatory subunits ( $\mathrm{p} 47^{\text {phox }}, \mathrm{p} 67^{\text {phox }}$, and Rac1) of Nox2 are localized to the 
T-tubule/sarcoplasmic reticulum junction. These subunits contribute to diastolic stretch-induced ROS production, followed by oxidative modification (activation) of ryanodine receptor 2 , leading to an increase in diastolic $\mathrm{Ca}^{2+}$ spark rate in the heart [24,25]. Additionally, transient receptor potential canonical (TRPC) 3 channel contributes to Nox2-mediated ROS production in rodent cardiomyocytes [26]. TRPC channels have two functions: they act as a sensor for mechanical stresses and as a protein scaffold at the plasma membrane to control the amplification and co-ordination of receptor signaling [27]. TRPC3 functionally interacts with PKC $\beta I I$ [28], and $\mathrm{Ca}^{2+}$-dependent activation of PKC $\beta \mathrm{II}$ may induce Nox2 activation through phosphorylation of the $\mathrm{p} 47^{\text {phox }}$ subunit [23]. Because PKC-mediated phosphorylation of Rho GDP dissociation inhibitor (GDI) $\alpha$ induces $\mathrm{Ca}^{2+}$-dependent Rac activation [29], TRPC3-mediated $\mathrm{Ca}^{2+}$ influx might dissociate RhoGDI $\alpha$ from Rac through PKC $\beta I I-d e p e n d e n t$ phosphorylation of RhoGDI $\alpha$. Therefore, functional interaction between TRPC3 and Nox2 may be involved in mechano-chemo transduction in the heart.

Topologically, Nox isozymes, except for Nox4, generate $\mathrm{O}_{2}{ }^{-}$in the extracellular area [22]. Conversion of $\mathrm{O}_{2}^{-}$into $\mathrm{H}_{2} \mathrm{O}_{2}$ by SOD is suggested to be essential for initiating ROS signaling. $\mathrm{H}_{2} \mathrm{O}_{2}$ might simply diffuse into the cell across the plasma membrane. However, recent studies suggest that $\mathrm{H}_{2} \mathrm{O}_{2}$ preferentially enters the cell through aquaporin channels on the plasma membrane [30,31]. Several ROS target proteins have been identified, including Trx [22], Nrf-2 [33], TRP [34,35], protein tyrosine phosphatase [36], and GTP-binding proteins (G proteins) [37]. These proteins contain particular Cys residues at active or allosteric sites, which are susceptible to oxidative modification, leading to regulation of specific downstream signaling pathways. Methionine is also a target amino acid for ROS. Oxidation of methionine on $\mathrm{Ca}^{2+} /$ calmodulin-dependent kinase II contributes to angiotensin (Ang) II-induced cardiac dysfunction in mice $[38,39]$. The reversibility of these oxidative modifications was confirmed by the discovery of their specific reductases, Trx reductase, GSH reductase, and methionine sulfoxide reductase [1].

\subsection{Formation, function and metabolism of 8-nitro-cGMP}

One of the major endogenous electrophiles is 8-nitro-cGMP, originally identified by Akaike's research group [40,41]. Formation of 8-nitro-cGMP requires RNS, such as peroxynitrite $\left(\mathrm{ONOO}^{-}\right)$generated from Nox2-derived ROS and inducible NOS (iNOS)-derived NO. More recently, mitochondria-derived superoxide production regulated by $\mathrm{H}_{2} \mathrm{O}_{2}$ generated from Nox2 was found to be essential for 8-nitro-cGMP 
formation in cells [42]. Using a stable isotope dilution technique and liquid chromatography coupled with tandem mass spectrometry analysis, more than $40 \mu \mathrm{M}$ of 8-nitro-cGMP was formed in cells, and these levels were much higher than cGMP levels formed in the same cells. This result indicates that 8-nitro-cGMP is one of the major cyclic nucleotides formed in cells. Additionally, 8-nitro-cGMP is not formed by direct nitration of cGMP, but is eventually generated after nitration of abundant GTP. This results in production of 8-nitro-GTP, which then reacts with guanylate cyclases to generate 8-nitro-cGMP. Among various guanine nucleotides, GTP is most susceptible to $\mathrm{ONOO}^{-}$-mediated nitration [43]. 8-Nitro-GTP formed by the reaction of GTP and $\mathrm{ONOO}^{-}$appears to be an efficient substrate for soluble guanylate cyclases to form 8-nitro-cGMP [44]. In fact, the electrophilicity of 8-nitro-cGMP is much lower than that of other endogenous electrophiles. The second-order rate constant for the reaction of 8-nitro-cGMP with GSH sulfhydryl is $0.03 \mathrm{M}^{-1} \mathrm{~s}^{-1}$ at $\mathrm{pH} 7.4$ and $37^{\circ} \mathrm{C}[41,45]$. Therefore, 8-nitro-cGMP is believed to be much less reactive compared with certain other electrophiles, such as $\alpha, \beta$-unsaturated aldehydes, $\omega-6$, and $\omega$-3 unsaturated fatty acids, and nitroalkene fatty acids. These nitroalkene fatty acids include 4-hydroxy-2-nonenal (HNE), 15-deoxy- $\Delta^{12,14}$-prostaglandin $\mathrm{J}_{2} \quad$ (15d-PGJ $\mathrm{J}_{2}$ ), and nitro-linoleic and nitro-oleic acids. The second-order rate constants for the reaction of those electrophiles with GSH are $1.3 \mathrm{M}^{-1} \mathrm{~s}^{-1}$ (HNE), $0.7 \mathrm{M}^{-1} \mathrm{~s}^{-1}$ (15d-PGJ $), 355 \mathrm{M}^{-1}$ $\mathrm{s}^{-1}$ (nitro-linoleic acid), and $183 \mathrm{M}^{-1} \mathrm{~s}^{-1}$ (nitro-oleic acid) at $\mathrm{pH} 7.4$ and $37^{\circ} \mathrm{C}$ [45-47]. These reactive electrophiles react readily with GSH and thus can be scavenged by GSH sulfhydryls more than 20-10,000 times more rapidly than by 8-nitro-cGMP.

Reactive electrophiles produced in cells during oxidative stress are detoxified by the actions of glutathione $S$-transferase (GST) [48]. However, 8-nitro-cGMP is not metabolized by GSH and is stable enough to remain at appreciable levels in cells, even with excessive amounts of GSH. Based on the fact that Cys sulfhydryls react with 8-nitro-cGMP, we found a considerable effect of endogenous hydrogen sulfide-related reactive molecules on 8-nitro-cGMP metabolism. Two important enzymes involved in reactive sulfur biosynthesis, cystathionine $\beta$-synthase (CBS) and cystathionine $\gamma$-lyase, mediate the metabolism of cellular 8-nitro-cGMP in various cultured mammalian cells. The electrophilic nitro moiety undergoes nucleophilic substitution, yielding the novel product 8-SH-cGMP (Figs. 1 and 2). This metabolism occurs concomitantly with the release of nitrite to generate 8 -SH-cGMP. This suggests a mechanism that depends on the nucleophilic qualities of $\mathrm{HS}^{-}$-like molecular species that predominate in neutral biological solutions. Similar electrophile sulfhydration occurs with a broad array of biological electrophiles. The metabolic fate of these sulfhydrated derivatives allows the 
sorting of electrophiles into three groups (Fig. 1). In the first group (including 8-nitro-cGMP and $15 \mathrm{~d}-\mathrm{PGJ}_{2}$ ), the sulfhydrated derivative is so stable that no additional reaction occurs, except for oxidative degradation of $\mathrm{SH}$ by ROS and other reactive species. The second group (including nitro-oleic acid, 1,2-naphthoquinone (1,2-NQ), 1,4-naphthoquinone, tert-butylbenzoquinone, $N$-ethylmaleimide, diethylmaleate, and monobromobimane) involves relatively stable bis product formation. The third group may include other highly reactive electrophiles, such as HNE and acrolein, for which additional metabolism and secondary chemical reactions follow SH addition. The end product 8-SH-cGMP appears to be extremely stable among sulfhydrated derivatives of the above-mentioned electrophiles. 8-SH-cGMP contains sulfhydryls but the sole product observed by further reaction with $\mathrm{H}_{2} \mathrm{O}_{2}$ was cGMP, rather than oxidized derivatives of 8-SH-cGMP, such as 8-sulfenyl-, 8-sulfinyl-, and 8-sulfonate-cGMP, or 8-OH-cGMP [14]. This result provides the first demonstration of oxidant-induced desulfhydration of $\mathrm{SH}$-containing compounds. All these findings suggest that reactive sulfur species produced in cells are a critical factor in 8-nitro-cGMP metabolism.

8-Nitro-cGMP undergoes nucleophilic substitution with a protein sulfhydryl, releases the nitro group, and forms a protein Cys-cGMP adduct in a process called protein S-guanylation. Several ROS sensor proteins, such as Keap1 and H-Ras, are modified (S-guanylated) by 8-nitro-cGMP [14,41]. More interestingly, S-guanylated bacteria are selectively marked with Lys63-linked polyubiquitin, a known molecular tag for selective transport to autophagosomes [49]. This autophagic protein degradation may be an alternative 8-nitro-cGMP metabolic pathway.

\subsection{Redox control of vascular tone}

Vascular endothelium is a major modulator of vascular tone, releasing vasoactive substances, such as endothelium-derived relaxing factor (EDRF), endothelium-derived hyperpolarizing factors (EDHFs), cycloxygenase metabolites, and endothelium-derived constricting factors [50,51]. Nobel laureates Furchgott, Murad, and Ignarro discovered $\mathrm{NO}$ as a major EDRF. NO induces vasodilation through cGMP-dependent protein kinase (PKG)-mediated phosphorylation of myosin light chain kinase in vascular smooth muscle cells. Another major EDRF is prostacyclin, which induces vasodilation through cAMP-dependent protein kinase-mediated phosphorylation of myosin light chain kinase. Accumulating evidence has shown that the contribution of EDRF in large arteries is higher than that in small arteries, while EDHF rather than EDRF contributes to vasodilation in smaller arteries [52]. Since the first report on the existence of EDHF, several substances/mechanisms have been 
proposed for the nature of EDHF, including epoxyeicosatrienoic acids, $\mathrm{K}^{+}$, and electrical communications through myoendothelial gap junctions. Additionally, endothelium-derived $\mathrm{H}_{2} \mathrm{O}_{2}$ via the endothelial NOS system, which is functionally coupled with $\mathrm{Cu}, \mathrm{Zn}-\mathrm{SOD}$, has been reported to work as an EDHF in mammalian coronary arteries [52]. Although it is still unclear whether endothelial NOS-derived $\mathrm{H}_{2} \mathrm{O}_{2}$ actually functions as an EDHF, $\mathrm{H}_{2} \mathrm{O}_{2}$ has been reported to induce vasodilation via oxidative activation of protein kinase and PKG [53-55]. Likewise, it has pharmacologically been shown that hydrogen sulfide, exogenously introduced, can exhibit vasodilatory effects indirectly by delaying cGMP degeneration through phosphodiesterase 5 inhibition [56] or ATP-sensitive $\mathrm{K}^{+}$channel activation [57]. However, hydrogen sulfide may also trigger dose-dependent vasoconstriction or vasodilatory effects depending on the type of vessel and the animal species examined. This conditional response toward hydrogen sulfide is suggested to be mediated via covalent modification of $\mathrm{K}^{+}$channel protein thiols or due to nitrosothiol formed as a result of NO/hydrogen sulfide interaction [58]. 8-Nitro-cGMP also shows dose-dependent vasoconstriction or vasodilatory effects [59]. Considering the nucleophilic property of hydrogen sulfide and its related reactive sulfur compounds, formation of 8-SH-cGMP via its nucleophilic substitution with reactive sulfur species is suggested to contribute to the vasodilatory effect of hydrogen sulfide because 8-SH-cGMP also has a potent vasodilatory activity (Fig. 2).

\section{Redox control of $\mathbf{G}$ protein signaling}

\subsection{G proteins and cardiovascular remodeling}

Cardiovascular remodeling is a process induced by common cardiovascular risk factors, such as high blood pressure, hyperglycemia, overweight/obesity, and smoking, which combine to modify the functional and structural characteristics of the myocardium and blood vessels. Cardiovascular remodeling includes a preclinical stage, during which symptoms are not evident. Cardiovascular remodeling also includes a clinical disease stage, which corresponds to the progression of preclinical disease and to the action of additional triggering mechanisms representing obvious tissue damage. Several neurohumoral factors, such as Ang II and noradrenaline, and their corresponding G protein-coupled receptors (GPCRs) have been implicated in the development of cardiovascular remodeling [60]. In most cases, protein expression levels of small G proteins, Ras, Rho, and Rac, and vasoconstrictive GPCRs (e.g., Ang type 1 receptor $\left[A T_{1} R\right]$ ) are significantly increased in the heart with heart failure [61]. Interestingly, recent studies have shown that the activities of $G$ proteins and their 
regulators, especially guanine nucleotide exchange factors and GTPase-activating proteins (GAPs), are functionally controlled by ROS and/or ROS/RNS-derived electrophiles through PTM mechanisms.

\subsection{Redox control of $G$ protein cycling}

$\mathrm{G}$ proteins function as binary molecular switches to regulate a wide range of cellular processes [62]. There are two classes of $G$ proteins. The first class functions as monomeric small $G$ proteins, while the second functions as heterotrimeric $G$ protein complexes. The latter class of complexes is composed of $\alpha, \beta$, and $\gamma$ subunits. The $\beta$ and $\gamma$ subunits can form a stable dimeric complex referred to as the $\beta \gamma$ complex. The conformation switch between the GDP-bound state and GTP-bound state is called the "G (protein) cycle" (Fig. 3). The exchange of GTP for GDP on G proteins is predominantly regulated by guanine nucleotide exchange factors and GAPs, which are originally believed to regulate the activation state of $G$ proteins without covalently modifying them. Several studies have shown that PTM (especially oxidative modification) directly or indirectly modulates $G$ cycle balance [63-66]. An example of this situation is where the protein expression level of regulator of $G$ protein signaling type 4 (RGS4), which is a major GAP for an $\alpha$ subunits of heterotrimeric $G_{q}$ and $G_{i}$ proteins among more than 20 RGS proteins, is negatively regulated by NO. This mechanism is termed "Arg/N-end rule", where $S$-nitrosylation of the N-terminal Cys residue, which is targeted for ubiquitin-dependent degradation by arginylation, promotes arginylation-dependent protein degradation via Cys oxidation [65,67,68]. This mechanism also participates in cardiovascular development [67] and placental growth factor-induced myocardial angiogenesis and hypertrophic growth of cardiomyocytes through endothelial NOS-dependent NO release [68].

Heterotrimeric $\mathrm{G}$ proteins located within the cell are activated by GPCRs that span the cell membrane. Therefore, GPCR and G proteins working together transmit signals from many hormones, neurotransmitters, and other signaling factors. The $\mathrm{AT}_{1} \mathrm{R}$, one of the guanine nucleotide exchanging factors for heterotrimeric $G_{q}, G_{i}$, and $G_{12}$ family proteins, contributes to various cardiovascular functions and diseases [60]. $\mathrm{AT}_{1} \mathrm{R}$ activity is negatively regulated by oxidation or reduction of $\mathrm{AT}_{1} \mathrm{Rs}[69,70]$. Disulfide bridges between the $\mathrm{N}$-terminal region and the third extracellular loop, and between the first and second extracellular loops, are formed to maintain the conformation of $\mathrm{AT}_{1} \mathrm{R}$ protein. $\mathrm{AT}_{2} \mathrm{R}$ is resistant to inactivation by reducing agents because of the absence of disulfide bridges between the N-terminal region and the third extracellular loop. Nitro-oleic acid binds to $\mathrm{AT}_{1} \mathrm{R}$, and reduces $\mathrm{G}_{\mathrm{q}}$ protein-mediated coupling without 
affecting Ang II binding to the receptor [71].

ROS and RNS also regulate $\mathrm{AT}_{1} \mathrm{R}$ transcriptional activity via PTM of a redox-sensitive transcriptional factor, nuclear factor- $\mathrm{B}$ [72]. We found that endogenous ROS generated by Pertusis toxin stimulation on Toll-like receptor 4 increases $\mathrm{AT}_{1} \mathrm{R}$ density in rat cardiac fibroblasts [73]. Toll-like receptor 4 stimulation by Pertusis toxin activates NADPH oxidase through Rac, a small G protein, and subsequently induces nuclear factor- $\kappa \mathrm{B}$ activation through ROS-dependent I $\mathrm{I} \mathrm{B} \alpha$ phosphorylation and degradation. In contrast, NO and nitro-unsaturated fatty acids reportedly decrease Ang II signaling in the heart $[71,74]$. Cys-38 of nuclear factor- $\kappa B$ wedged between acidic and basic amino acids is nucleophilic, acidic, and redox active, and numerous reactions may occur on this Cys thiol side chain. The reactive Cys located in the Rel homology domain is involved in DNA binding or dimer formation of p65. NO-mediated Cys38 modification (S-nitrosylation) of p65 is expected to decrease transcriptional activity of nuclear factor- $\kappa \mathrm{B}$. This mechanism includes formation of a protein signaling complex among iNOS, p65, and $\beta$-arrestin2 around the perinuclear region. Therefore, the spatiotemporal regulation of Cys modification by ROS or RNS may underlie the resultant changes of $\mathrm{AT}_{1} \mathrm{R}$ signaling.

\subsection{Regulation of G protein activity by PTM}

The first two decades of research on the redox control of $G$ protein signaling were developed by the understanding of how oxidative PTM enhances the exchange of GTP for GDP in Ras family $G$ proteins. Many $G$ proteins have reactive Cys residues in the guanine nucleotide-binding domain of GTPase (Fig. 4). The monomeric Ras family G proteins commonly possess Cys residues in the center (NKCD) or in the phosphate-binding loop (P-loop) of the guanine nucleotide-binding region $[64,66]$. Additionally, $\alpha$ subunits of heterotrimeric $\mathrm{G}_{\mathrm{i} / \mathrm{o}}$ proteins have reactive Cys residues at the $\alpha G / \alpha 4$ and $\beta 6 / \alpha 5$ loops located near the guanine nucleotide-binding region [75]. The modification of these Cys residues actually increases the dissociation rate of guanine nucleotide, while this modification does not necessarily increase GTP-binding activity [76]. In fact, thioredoxin-1-sensitive oxidative modification or S-glutathionylation of Ras mediates $\alpha$-adrenergic receptor-stimulated or mechanical strain-induced hypertrophy in adult rat ventricular myocytes $[77,78]$. This mechanism is fundamentally explained by the modification of Cys-118, a critical reactive Cys located in the guanine nucleotide-binding site. In contrast, Raines et al. reported that nNOS-mediated S-nitrosylation of $\mathrm{H}$-Ras at Cys-118 suppresses $\mathrm{H}$-Ras activity induced by $\mathrm{Ca}^{2+}$ ionophores [76]. We have also found that modification of Cys-118 is not stable and 
gradually decreases GTPase activity of recombinant H-Ras (unpublished observation). Therefore, it appears that modification of Cys-118 on Ras transiently, but not continuously, activates Ras-dependent signaling pathways.

Recent studies have shown that PTM of G proteins, especially monomeric small G proteins, direct them to various cellular membranes and modulate GTP-GDP exchange [79]. Important PTM of G proteins includes the constitutive and irreversible remodeling of its carboxy-terminal CAAX motif. In this remodeling in which C is Cys, A is usually an aliphatic amino acid, and $\mathrm{X}$ is any amino acid, by farnesylation (isoprenylation), proteolysis and methylation, reversible palmitoylation, and conditional modifications, including phosphorylation, peptididyl-prolyl isomerization, mono- and di-ubiquitination, ADP ribosylation, and glycosylation. One of two palmitoylation sites (Cys-184) of H-Ras has been shown as a redox-active Cys. Oxidative modification of Cys proximal to the CAAX motif suppresses or in some cases accelerates other PTMs, leading to modification of $\mathrm{G}$ protein activity [80,81].

\section{Redox control of cardiac remodeling}

\subsection{Formation of 8-nitro-cGMP in cardiac cells.}

Chronic heart failure is a leading cause of morbidity and mortality in developed countries. ROS and NO generated from Nox2 and iNOS have been implicated in the pathogenesis of heart failure. In fact, 3-nitrotyrosine, an endogenous nitrating product, is actually generated in mouse hearts after myocardial infarction (MI), accompanied by a dramatic increase in expression levels of Nox2 and iNOS proteins. Using liquid chromatography coupled with tandem mass spectrometry analysis and immunohistochemistry with an anti-8-nitro-cGMP specific antibody, we showed that 8-nitro-cGMP is produced in mouse hearts 4 weeks after MI and 6 weeks after pressure overload [14]. Inducible NOS-derived NO may be essential for 8-nitro-cGMP formation in mouse hearts because 8-nitro-cGMP formation after MI is completely diminished in iNOS-deficient hearts. Although protein expression levels of iNOS, but not Nox2, are promptly increased in mouse hearts after MI, 8-nitro-cGMP formation is not present in mouse hearts within 1 week after MI. These results indicate that mouse hearts produce 8-nitro-cGMP in response to ischemia or mechanical stress in an iNOS-dependent manner, while Nox2-derived ROS may be essential for the formation of 8-nitro-cGMP by iNOS-derived NO [14].

\subsection{Cardiac cell senescence via S-guanylation of $\mathbf{H}$-Ras}

Cardiac aging is one of the major causes of sudden death or left ventricular 
dysfunction after MI. Cellular senescence is reportedly induced by oxidative stress, nucleotide damage (e.g., UV irradiation), and abnormal activation of oncogenes, including Ras family $G$ proteins [82,83]. Recent studies of oxidative inflammatory reactions suggest that an $\mathrm{H}$-Ras oncogenic cellular response can be induced by ROS- or RNS-derived species and 15d-PGJ 2 [64,84], which in turn may activate p53-dependent cellular senescence. We found that 8-nitro-cGMP mediates cardiac cellular senescence via $S$-guanylation of H-Ras at Cys-184 in failing hearts in mice after MI. In contrast, another electrophile, 1,2-NQ, does not modify Cys-184 of H-Ras or induce H-Ras activation, although 1,2-NQ strongly increases ERK activity in cardiomyocytes [14]. Among several endogenous and exogenous electrophiles, 8-nitro-cGMP is suggested to function as a specific physiological ligand for H-Ras activation. H-Ras possesses two palmitoylation sites (Cys-181 and Cys-184) located at its carboxy-terminal domain, and palmitoylation of Ras proteins plays an important role in Ras protein localization and activity [80]. Monopalmitoylation of Cys-181 is required for efficient trafficking of $\mathrm{H}$-Ras to the plasma membrane, and GDP-bound H-Ras is predominantly detected in lipid rafts [80]. GTP loading of H-Ras releases H-Ras from the rafts so that they become more diffusely distributed in the plasma membrane, an event necessary for efficient activation of Raf. Although Cys-184 is not essential for targeting of H-Ras to the plasma membrane, it is essential for control of GTP-regulated lateral segmentation of H-Ras between lipid rafts and non-rafts. Inhibition of Cys-184 by 8-nitro-cGMP palmitoylation efficiently delivers H-Ras to the plasma membrane. This indicates that $S$-guanylation of $\mathrm{H}$-Ras at Cys-184 promotes localization of $\mathrm{H}$-Ras to the plasma membrane and association with Raf by dissociating $\mathrm{H}$-Ras from lipid rafts. Oxidative stress-related nucleotides that induce DNA and nucleotide damage, as well as activation of oncogenic Ras, can induce cellular senescence via p53 and Rb tumor suppressor pathways [82,83]. Our recent findings provide evidence that electrophilic modification causes precise structural alterations of H-Ras, making it more accessible to Raf [14]. This leads to transduction of electrophile-mediated signaling to downstream phosphorylation signaling, including extracellular signal-regulated kinase, p38 mitogen-activated protein kinase, p53, and Rb.

\subsection{Prevention of cardiac senescence via elimination of electrophiles by reactive sulfur species}

Although endogenous hydrogen sulfide is thought to be formed by CBS and cystathionine $\gamma$-lyase in mammalian cells and tissues [58], the exact chemical nature and physiological functions of the products of their enzymatic reaction are mostly undefined. 
CBS knockdown significantly suppresses generation of reactive sulfur metabolites, which are responsible for electrophile sulfhydration as discussed above, in several tumor cell lines (A549, HepG2, and C6 cells) expressing abundant CBS proteins [14]. In contrast, lower levels of CBS and cystathionine $\gamma$-lyase expression as well as a much lower production of reactive sulfur metabolites are observed in mouse hearts, primary-cultured rat neonatal cardiomyocytes, and cardiac fibroblasts compared with all tumor cell lines. Therefore, reactive sulfur metabolites in the heart may be limiting in the context of the amount of endogenous electrophiles that can be generated during basal metabolism and inflammatory responses. Therefore, myocardial cells may be relatively sensitive to endogenous electrophiles, particularly when they are excessively generated during inflammatory processes.

We evaluated pharmacological activities of hydrogen sulfide derivatives in an in vivo model of chronic heart failure by exogenous treatment with NaHS [14]. Considerable 8-nitro-cGMP production, which depends on iNOS expression and activity, is strongly inhibited by low-dose NaHS (50 $\mu \mathrm{mol} / \mathrm{kg} /$ day, i.p.) in mouse hearts after MI. NaHS treatment has no significant effects on tyrosine nitration reaction occurring in cardiac tissues, suggesting that hydrogen sulfide does not directly scavenge ROS and RNS, but other related sulfur species might eliminate ROS/RNS, and even their derived electrophiles. The metabolic pathways related to cGMP biosynthesis and degradation (e.g., sGC and PDEs) are not affected by the same treatment.

NaHS treatment fully inhibits H-Ras activation and concomitant H-Ras S-guanylation in post-MI mouse hearts. NaHS treatment in vivo after MI significantly improves left ventricular dilation and limits left ventricular dysfunction in mice, although ischemic scars are equally developed in hearts of NaHS- and vehicle-treated mice [14]. Furthermore, phosphorylation of p38 mitogen-activated protein kinase, extracellular signal-regulated kinase, p53, and Rb protein is increased in mouse hearts after MI, with NaHS suppressing their activation. These results confirm that any particular reactive sulfur metabolites attenuate left ventricular dilation and dysfunction after MI, in part by suppressing myocardial cell $S$-guanylation-dependent activation of H-Ras and its downstream signaling pathways.

\section{Conclusions}

Although 8-nitro-cGMP is a moderate electrophile compared with other electrophiles generated by ROS, 8-nitro-cGMP functions as a newly discovered important player in the development of heart failure. ROS signaling is precisely regulated by various sensor and effector molecules, and the unique property of 8-nitro-cGMP appears to contribute 
to its specificity and selectivity for ROS signal transduction. The emergence of new evidence supporting the role of electrophilic modifications of Ras family proteins may presage a new era in cardiovascular biology. H-Ras knockout mice show hypotension via enhancing the NO-cGMP-PKG pathway [85] and R-Ras knockout mice show abnormal maturation of new blood vessels in tumors [86]. Therefore, further extensive in vivo studies focusing on S-guanylation induced by 8-nitro-cGMP may reveal new aspects of redox control of cardiovascular physiological functions, such as vascular tone and angiogenesis. Notably, our recent investigation revealed that ROS and electrophiles were primarily regulated by reactive sulfur species such as Cys hydropersulfide (CysSSH) and its derivatives, endogenously occurring in most tissues and cells, rather than other standard sulfur-containing metabolites like glutathione and hydrogen sulfide (unpublished observation). Control of electrophilic PTM using nucleophilic properties of reactive sulfur compounds could be a new therapeutic strategy for the prevention and treatment of chronic cardiovascular diseases.

\section{Funding sources}

This work was supported in part by Grants-in-Aid for Scientific Research and Grants-in-Aid for Scientific Research on Innovative Areas (Research in a Proposed Research Area), from the Ministry of Education, Culture, Sports, Science and Technology. This work was also supported by PRESTO, Japan Science and Technology Agency, and the Tokyo Biochemical Research Foundation. 
[1] Finkel T. Signal transduction by reactive oxygen species. J Cell Biol 2011; 194:7-15.

[2] Lambert JD. NOX enzymes and the biology of reactive oxygen. Nature Rev Immunol 2004; 4:181-9.

[3] Rosca MG, Tandler B, Hoppel. Mitochondria in cardiac hypertrophy and heart failure. J Mol Cell Cardiol 2013; 55:31-41.

[4] Burgoyne JR, Oka S, Ale-Agha N, Eaton P. Hydrogen peroxide sensing and signaling by protein kinases in the cardiovascular system. Antioxid Red Signal 2013; 18:1042-52.

[5] Stanley BA, Sivakumaran V, Shi S, McDonald I, Lloyd D, Watson WH. Thioredoxin reductase-2 is essential for keeping low levels of $\mathrm{H}_{2} \mathrm{O}_{2}$ emission from isolated Heart Mitochondria. J Biol Chem 2011 286:33669-77.

[6] Woo HA, Yim SH, Shin DH, Kang D, Yu DY, Rhee SG. Inactivation of peroxiredoxin I by phosphorylation allows localized $\mathrm{H}_{2} \mathrm{O}_{2}$ accumulation for cell signaling. Cell 2010; 140:517-28.

[7] McCann JC, Ame BN. Adaptive dysfunction of selenoproteins from the perspective of the triage theory: why modest selenium deficiency may increase risk of diseases of aging. FASEB J 2011; 25:1793-1814.

[8] de Lorgeril M, Salen P. Selenium and antioxidant defenses as major mediators in the development of chronic heart failure. Heart Fail Rev 2006; 11:13-7.

[9] Zhang Y, Ikeno Y, Qi W, Chaudhuri A, Li Y, Bokov A et al. Mice deficient in both Mn superoxide dismutase and glutathione peroxidase- 1 have increased oxidative damage and a greater incidence of pathology but no reduction in longevity. $J$ Gerontol A Biol Sci Med Sci 2009; 64:1212-20.

[10] Nagy N, Malik G, Fisher AB, Das DK. Targeted disruption of peroxiredoxin 6 gene renders the heart vulnerable to ischemia-reperfusion injury. Am J Physiol Heart Circ Physiol 2006; 291:H2636-40.

[11] Tosaki A, Edes I. The role of peroxiredoxins in ischemia-reperfusion-induced cardiac damage. Am J Physiol Heart Circ Physiol 2006; 291:H2586-7.

[12] Huang TT, Carlson EJ, Kozy HM, Mantha S, Goodman SI, Ursell PC, et al. Genetic modification of prenatal lethality and dilated cardiomyopathy in $\mathrm{Mn}$ superoxide dismutase mutant mice. Free Radic Biol Med 2001; 31:1101-10.

[13] Rudolph TK, Freeman BA. Transduction of redox signaling by electrophile-protein reactions. Sci Signal 2009; 2(90):re7. doi: 10.1126/scisignal.290re7

[14] Nishida M, Sawa T, Kitajima N, Ono K, Inoue H, Ihara H et al. Hydrogen sulfide anion regulates redox signaling via electrophile sulfhydration. Nat Chem Biol 2012; 8:714-24. 
[15] Yang G, Wu L, Jiang B, Yang W, Qi J, Cao K et al. H2S as a physiologic vasorelaxant: hypertension in mice with deletion of cystathionine $\gamma$-lyase. Science 2008; 322:587-90.

[16] Mustafa AK, Sikka G, Gazi SK, Steppan J, Jung SM, Bhunia AK, et al. Hydrogen sulfide as endothelium-derived hyperpolarizing factor sulfhydrates potassium channels. Circ Res 2011; 109:1259-68.

[17] Morikawa T, Kajimura M, Nakamura T, Hishiki T, Nakanishi T, Yukutake Y, et al. Hypoxic regulation of the cerebral microcirculation is mediated by a carbon monoxide-sensitive hydrogen sulfide pathway. Proc Natl Acad Sci U S A 2012; 109:1293-8.

[18] Saito Y, Berk BC. Angiotensin II-mediated signal transduction pathways. Curr Hypertens Rep 2002; 4:L167-L171.

[19] Touyz RM. Reactive oxygen species as mediators of calcium signaling by Angiotensin II: implications in vascular physiology and pathophysiology, Antioxid Redox Signal 2005; 7:1302-1314.

[20] Rajasekaran NS, Connell P, Christians ES, Yan LJ, Taylor RP, Orosz A, et al. Human alpha B-crystallin mutation causes oxido-reductive stress and protein aggregation cardiomyopathy in mice. Cell 2007; 130:427-39.

[21] Dimmeler S, Zeiher AM. A "reductionist" view of cardiomyopathy. Cell 2007; 130:401-2.

[22] Sumimoto H. Structure, regulation and evolution of Nox-family NADPH oxidases that produce reactive oxygen species. FEBS J 2008; 275: 3249-77.

[23] Brown GE, Stewart MQ, Liu H, Ha V-L, Yaffe MB. A novel assay system implicates PtdIns(3,4)P2, PtdIns(3)P, and PKC $\delta$ in intracellular production of reactive oxygen species by the NADPH oxidase. Mol Cell 2003; 11:35-47.

[24] Prosser BL, Ward CW, Lederer WJ. X-ROS signaling: rapid mechano-chemo transduction in heart. Science 2011; 333:1440-5.

[25] Patel VB, Wang Z, Fan D, Zhabyeyev P, Basu R, Das SK et al. Loss of p47 $7^{\text {phox }}$ subunit enhances susceptibility to biomechanical stress and heart failure because of dysregulation of cortactin and actin filaments. Circ Res 2013; 112:1542-56.

[26] Kitajima N, Watanabe K, Morimoto S, Sato Y, Kiyonaka S, Hoshijima M et al. TRPC3-mediated $\mathrm{Ca}^{2+}$ influx contributes to Rac1-mediated production of reactive oxygen species in MLP-deficient mouse hearts. Biochem Biophys Res Commun 2011; 409:108-13.

[27] Nishida M, Hara Y, Yoshida T, Inoue R, Mori Y. TRP channels: Molecular diversity and physiological function. Microcirculation 2006; 13:535-50. 
[28] Numaga T, Nishida M, Kiyonaka S, et al. $\mathrm{Ca}^{2+}$ influx and protein scaffolding via TRPC3 sustain PKC $\beta$ and ERK activation in B cells. J Cell Sci 2010; 123:927-38.

[29] Price LS, Langeslag M, ten Klooster JP, Hordijk PL, Jalink K, Collard JG. Calcium signaling regulates translocation and activation of Rac. J Biol Chem 2003; 278:39413-21.

[30] Bienert GP, Møller AL, Kristiansen KA, Schulz A, Møller IM, Schjoerring JK, et al. Specific aquaporins facilitate the diffusion of hydrogen peroxide across membranes. J Biol Chem 2007; 282:1183-92.

[31] Miller EW, Dickinson BC, Chang CJ. Aquaporin-3 mediates hydrogen peroxide uptake to regulate downstream intracellular signaling. Proc Natl Acad Sci USA 2010; 107:15681-86.

[32] Saitoh M, Nishitoh H, Fujii M, Takeda K, Tobiume K, Sawada Y, et al. Mammalian thioredoxin is a direct inhibitor of apoptosis signal-regulating kinase (ASK) 1. EMBO J 1998; 17:2596-606.

[33] Itoh K, Chiba T, Takahashi S, Ishii T, Igarashi K, Katoh Y, et al. An Nrf2/small Maf heterodimer mediates the induction of phase II detoxifying enzyme genes through antioxidant response elements. Biochem Biophys Res Commun 1997; 236:313-22.

[34] Yoshida T, Inoue R, Morii T, Takahashi N, Yamamoto S, Hara Y, et al. Nitric oxide activates TRP channels by cysteine S-nitrosylation. Nat Chem Biol 2006; 2:596-607.

[35] Takahashi N, Kuwaki T, Kiyonaka S, Numata T, Kozai D, Mizuno Y, et al. TRPA1 underlies a sensing mechanism for O2. Nat Chem Biol 2011; 7: 701-11.

[36] Meng TC, Buckley DA, Galic S, Tiganis T, Tonks NK. Regulation of insulin signaling through reversible oxidation of the protein-tyrosine phosphatases TC45 and PTP1B. J Biol Chem 2004; 279:37716-25.

[37] Nishida M, Maruyama Y, Tanaka R, Kontani K, Nagao T, Kurose H. G $\alpha_{i}$ and $G \alpha_{o}$ are target proteins of reactive oxygen species. Nature 2000; 408:492-5.

[38] Erickson JR, Joiner ML, Guan X, Kutschke W, Yang J, Oddis CV, et al. A dynamic pathway for calcium-independent activation of CaMKII by methionine oxidation. Cell 2008; 133: 462-74.

[39] He BJ, Joiner ML, Singh MV, Luczak ED, Swaminathan PD, Koval OM, et al. Oxidation of CaMKII determines the cardiotoxic effect of aldosterone. Nat Med 2011; 17:1610-8.

[40] Sawa T, Zaki MH, Okamoto T, Akuta T, Tokutomi Y, Kim-Mitsuyama S, et al. Protein S-guanylation by the biological signal 8-nitroguanosine 3',5'-cyclic monophosphate. Nat Chem Biol 2007; 3:727-35. 
[41] Akaike T, Nishida M, Fujii S. Regulation of redox signalling by an electrophilic cyclic nucleotide. J Biochem 2013;153:131-8.

[42] Rahaman MM, Sawa $T$, Ahtesham AK, Khan $S$, Inoue $H$, Irie $A$, et al. S-guanylation proteomics for redox-based mitochondrial signaling. Antioxid Redox Signal 2012; doi:10.1089/ars.2012.4606.

[43] Ahmed KA, Sawa T, Ihara H, Kasamatsu S, Yoshitake J, Rahaman MM, et al. Regulation by mitochondrial superoxide and NADPH oxidase of cellular formation of nitrated cyclic GMP: potential implications for ROS signalling. Biochem J 2012; 441:719-30.

[44] Fujii S, Sawa T, Ihara H, Tong KI, Ida T, Okamoto T, et al. The critical role of nitric oxide signaling, via protein S-guanylation and nitrated cyclic GMP, in the antioxidant adaptive response. J Biol Chem 2010;285:23970-84.

[45] Sawa T, Arimoto H, Akaike T. Regulation of redox signaling involving chemical conjugation of protein thiols by nitric oxide and electrophiles. Bioconjug Chem 2010; 21:1121-9.

[46] Baker LM, Baker PR, Golin-Bisello F, Schopfer FJ, Fink M, Woodcock SR, et al. Nitro-fatty acid reaction with glutathione and cysteine. Kinetic analysis of thiol alkylation by a Michael addition reaction. J Biol Chem. 2007; 282:31085-93.

[47] Doorn JA, Petersen DR. Covalent adduction of nucleophilic amino acids by 4-hydroxynonenal and 4-oxononenal. Chem Biol Interact. 2003; 143-144:93-100.

[48] Hayes JD, Flanagan JU, Jowsey IR. Glutathione transferases. Annu Rev Pharmacol Toxicol 2005; 45:51-88.

[49] Ito C, Saito Y, Nozawa T, Fujii S, Sawa T, Inoue H, et al. Endogenous Nitrated Nucleotide Is a Key Mediator of Autophagy and Innate Defense against Bacteria. Mol Cell. 2013; 52:1-11.

[50] Vanhoutte, PM. Endothelium and control of vascular function. State of the Art lecture. Hypertension 1989; 13:658-67.

[51] Taddei S, Ghiadoni L, Virdis A, Versari D, Salvetti A. Mechanisms of endothelial dysfunction: clinical significance and preventive non-pharmacological therapeutic strategies. Curr Pharm Des 2003; 9:2385-402.

[52] Shimokawa H. Hydrogen peroxide as an endothelium-derived hyperpolarizing factor. Pflugers Arch. 2010; 459:915-22.

[53] Burgoyne JR, Madhani M, Cuello F, Charles RL, Brennan JP, Schröder E, et al. Cysteine redox sensor in PKGI $\alpha$ enables oxidant-induced activation. Science 2007; 317:1393-7.

[54] Prysyazhna O, Rudyk O, Eaton P. Single atom substitution in mouse protein kinase 
G eliminates oxidant sensing to cause hypertension. Nat Med 2012; 18:286-90.

[55] Brennan JP, Bardswell SC, Burgoyne JR, Fuller W, Schröder E, Wait R, et al. Oxidant-induced activation of type I protein kinase A is mediated by RI subunit interprotein disulfide bond formation. J Biol Chem 2006; 281:21827-36.

[56] Bucci M, Papapetropoulos A, Vellecco V, Zhou Z, Pyriochou A, Roussos C. Hydrogen sulfide is an endogenous inhibitor of phosphodiesterase activity. Arterioscler Thromb Vasc Biol 2010; 30:1998-2004.

[57] Zhao W, Zhang J, Lu Y, Wang R. The vasorelaxant effects of $\mathrm{H}_{2} \mathrm{~S}$ as a novel endogenous gaseous $\mathrm{K}_{\mathrm{ATP}}$ channel opener. EMBO J 2001; 20:6008-16.

[58] Kolluru GK, Shen X, Kevil CG. A tale of two gases: NO and H2S, foes or friends for life? Redox Biol 2013; 1:313-8.

[59] Tokutomi Y, Kataoka K, Yamamoto E, Nakamura T, Fukuda M, Nako H, et al. Vascular responses to 8-nitro-cyclic GMP in non-diabetic and diabetic mice. Brit J Pharmacol 2011; 162:1884-93.

[60] Sunggip C, Kitajima N, Nishida M. redox control of cardiovascular homeostasis by angiotensin II. Curr Pharm Des 2013; 19: 3022-32.

[61] Nishida M. Roles of heterotrimeric GTP-binding proteins in the progression of heart failure. J Pharmacol Sci. 2011; 117:1-5.

[62] Simon, M.I., Strathmann, M.P., \& Gautam, N. Diversity of G proteins in signal transduction. Science 1991; 252:802-8.

[63] Nishida M, Maruyama Y, Tanaka R, Kontani K, Nagao T, Kurose H. G $\alpha_{i}$ and G $\alpha_{0}$ are target proteins of reactive oxygen species. Nature 2000; 408:492-5.

[64] Lander HM, Milbank AJ, Tauras JM, Hajjar DP, Hempstead BL, Schwartz GD, et al. Redox regulation of cell signalling. Nature. 1996; 381:380-1.

[65] Madura K, Varshavsky A. Degradation of G alpha by the N-end rule pathway. Science. 1994; 265:1454-8.

[66] Heo J, Campbell SL. Mechanism of redox-mediated guanine nucleotide exchange on redox-active Rho GTPases. J Biol Chem. 2005; 280:31003-10.

[67] Kwon YT, Kashina AS, Davydov IV, Hu RG, An JY, Seo JW, et al. An essential role of N-terminal arginylation in cardiovascular development. Science 2002; 297:96-9.

[68] Jaba IM, Zhuang ZW, Li N, Jiang Y, Martin KA, Sinusas AJ, et al. NO triggers RGS4 degradation to coordinate angiogenesis and cardiomyocyte growth. J Clin Invest 2013; 123:1718-31.

[69] Domazet I, Holleran BJ, Martin SS, Lavigne P, Leduc R, Escher E, et al. The second transmembrane domain of the human type 1 angiotensin II receptor 
participates in the formation of the ligand binding pocket and undergoes integral pivoting movement during the process of receptor activation. J Biol Chem 2009; 284:11922-9.

[70] Domazet I, Martin SS, Holleran BJ, Morin ME, Lacasse P, Lavigne P, et al. The fifth transmembrane domain of angiotensin II type 1 receptor participates in the formation of the ligand-binding pocket and undergoes a counterclockwise rotation upon receptor activation. J Biol Chem 2009; 284:31953-61.

[71] Zhang J, Villacorta L, Chang L, Fan Z, Hamblin M, Zhu T, et al. Nitro-oleic acid inhibits angiotensin II-induced hypertension. Circ Res 2010; 107:540-8.

[72] Nishida M, Kitajima N, Saiki S, Nakaya M, Kurose H. Regulation of Angiotensin II receptor signaling by cysteine modification of NF-אB. Nitric Oxide 2011; 25:112-7.

[73] Nishida M, Suda R, Nagamatsu Y, Tanabe S, Onohara N, Nakaya M, et al. Pertussis toxin upregulates angiotensin type1 receptors through TLR4-mediated Rac activation. J Biol Chem 2010; 285:15268-77.

[74] Nishida M, Ogushi M, Suda R, Toyotaka M, Saiki S, Kitajima N, et al. Heterologous down-regulation of angiotensin type 1 receptors by purinergic $\mathrm{P}_{2} \mathrm{Y}_{2}$ receptor stimulation through S-nitrosylation of NF-кB. Proc Natl Acad Sci U S A 2011; 108:6662-7.

[75] Nishida M, Schey KL, Takagahara S, Kontani K, Katada T, Urano Y, et al. Activation mechanism of $G_{i}$ and $G_{o}$ by reactive oxygen species. J Biol Chem 2002; 277:9036-42.

[76] Raines KW, Cao GL, Lee EK, Rosen GM, Shapiro P. Neuronal nitric oxide synthase-induced S-nitrosylation of H-Ras inhibits calcium ionophore-mediated extracellular-signal-regulated kinase activity. Biochem J 2006; 397:329-36.

[77] Kuster GM, Pimentel DR, Adachi T, Ido Y, Brenner DA, Cohen RA, et al. $\alpha$-adrenergic receptor-stimulated hypertrophy in adult rat ventricular myocytes is mediated via thiredoxin-1-sensitive oxidative modification of thiols on Ras. Circulation 2005; 111:1192-8.

[78] Pimentel DR, Adachi T, Ido Y, Heibeck T, Jiang B, Lee Y, et al. Strain-stimulated hypertrophy in cardiac myocytes is mediated by reactive oxygen species-dependent Ras S-glutathionation. J Mol Cell Cardiol 2006; 41:613-22.

[79] Ahearn IM, Haigis K, Bar-Sagi D, Philips MR. Regulating the regulator: post-translational modification of Ras. Nat Rev Mol Cell Biol 2012; 13:39-51.

[80] Hancock JF. Ras Proteins: different signals from different locations. Nat Rev Mol Cell Biol 2003; 4:373-84. 
[81] Baker TL, Booden MA, Buss JE. S-Nitrosocysteine increases palmitate turnover on Ha-Ras in NIH 3T3 cells. J Biol Chem. 2000; 275:22037-47.

[82] Serrano M, Lin AW, McCurrach ME, Beach D, Lowe SW. Oncogenic ras provokes premature cell senescence associated with accumulation of p53 and p16 ${ }^{\mathrm{INK} 4 \mathrm{a}}$. Cell 1997; 88:593-602.

[83] Wu C, Miloslavskaya I, Demontis S, Maestro R, Galaktionov K. Regulation of cellular response to oncogenic and oxidative stress by Seladin-1. Nature 2004; 432:640-5.

[84] Oliva JL, Pérez-Sala D, Castrillo A, Martínez N, Cañada FJ, Boscá L, et al. The cyclopentenone 15-deoxy-delta 12,14-prostaglandin J2 binds to and activates H-Ras. Proc Natl Acad Sci U S A 2003; 100:4772-7.

[85] Chamorro-Jorganes A, Grande MT, Herranz B, Jerkic M, Griera M, Gonzalez-Nuñez, $M$ et al. Targeted genomic disruption of H-Ras induces hypotension through a NO-cGMP-PKG pathway-dependent mechanism. Hypertension 2010; 56:484-9.

[86] Sawada J, Urakami T, Li F, Urakami A, Zhu W, Fukuda M, et al. Small GTPase R-Ras regulates integrity and functionality of tumor blood vessels. Cancer Cell 2012; 22:235-49. 


\section{Figure legends}

Figure 1. Sulfhydration of electrophiles (E) by reactive sulfur speices and subsequent regulation of metabolic pathways. Reactive sulfur species that can behave like nucelophilic $\mathrm{HS}^{-}$act as a potent nucleophile and are thought to suppress cardiac senescence and dysfunction by inhibiting electrophilic modification of sensor proteins, including H-Ras.

Figure 2. Putative redox control of vascular tone. In small arteries, both electrophiles (i.e., 8-nitro-cGMP) and reactive sulfur function as vasodilators. However, nucleophilic reaction of $\mathrm{HS}^{-}$may quench 8-nitro-cGMP, leading to suppression of vasodilation mediated by 8-nitro-cGMP and reactive sulfurs.

Figure 3. Redox control of the G cycle. GAP; GTPase activating protein, GDI; GDP dissociation inhibitor, GEF; GTP/GDP exchange factor, PB; carboxy-terminal polybasic domain, SW; switch region.

Figure 4. Conserved reactive Cys residues in Ras family proteins. 


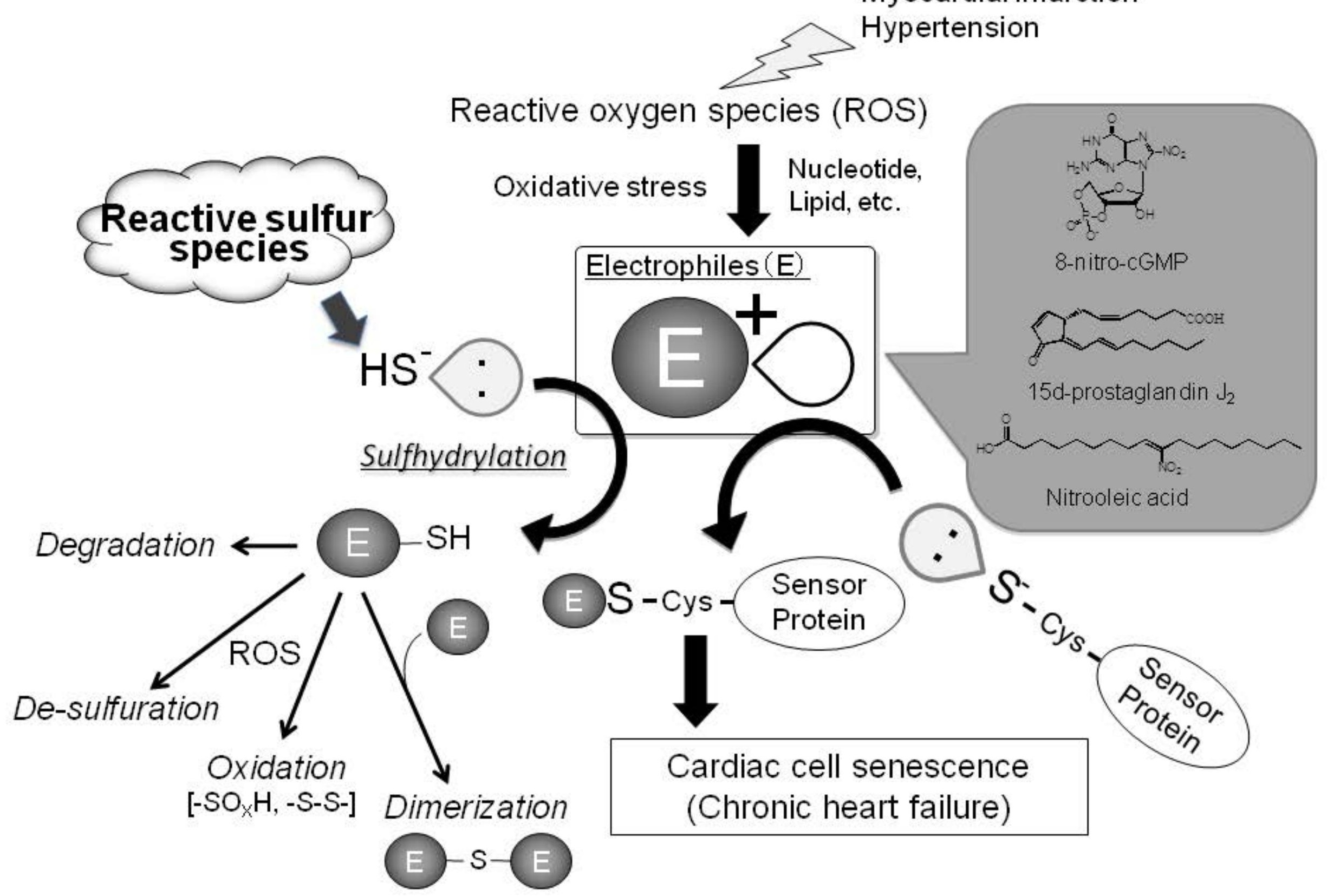


Figure 3

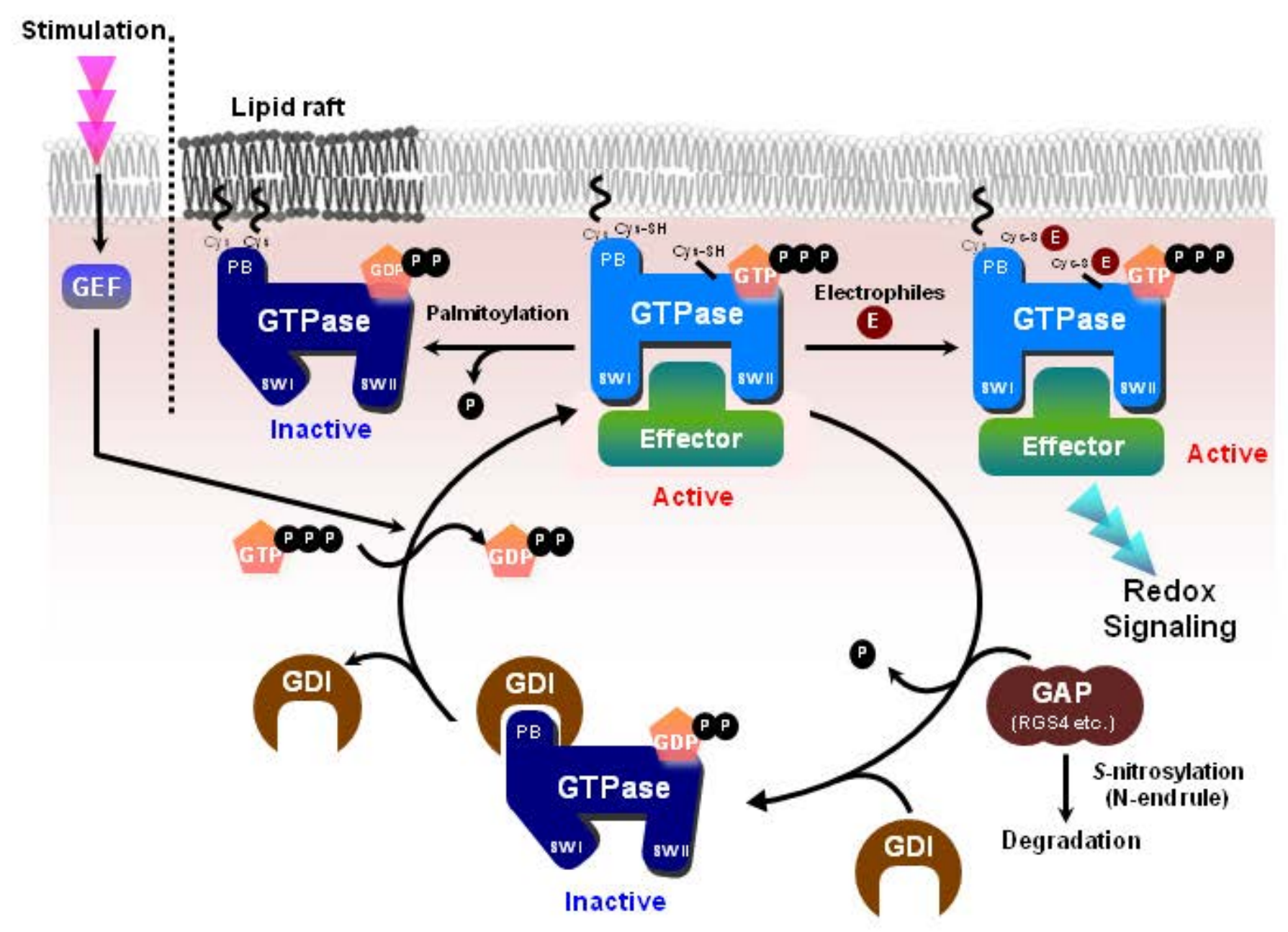


mouse H-Ras $\quad \ldots{ }^{111}$ MVLVGNKCDL $\cdots$

$\mathrm{N}$-Ras $\quad \ldots{ }^{111} \mathrm{MVLVGNKCDL} \cdots$

K-Ras $\quad \ldots{ }^{111}$ MVLVGNKCDL $\cdots$.

M-Ras $\quad \ldots{ }^{121}$ MILVANKVDL $\cdots$

Palmitoylation.${ }^{180}$ KSRTRCTVM

R-Ras $\quad \ldots{ }^{137}$ IVLVGNKADL $\cdots$

Rap1A $\ldots{ }^{111}$ MILVGNKCDL $\cdots$

Rap1B $\quad \ldots{ }^{111}$ MILVGNKCDL $\cdots$

Rap2A $\ldots{ }^{111}$ VILVGNKVDL $\cdots$

Rap2B $\quad \ldots{ }^{111}$ MILVGNKVDL $\cdots$

Rab1 $\quad .{ }^{119}$ KLLVGNKCDL $\cdots$

Rac1 $\quad . .{ }^{109}$ IILVGTKLDL $\cdots$

RhoB $\quad \ldots{ }^{112}$ IILVGNKKDL $\cdots$

$\ldots{ }^{201}$ THKLQCVIL

... ${ }^{211}$ DGGCPCVLL

... ${ }^{176}$ PKKKSSCLLL

$\ldots{ }^{176}$ RKKSSCQLL

... ${ }^{175}$ PCCSACNIQ

$\ldots{ }^{175} \mathrm{GCCSACVIL}$

... ${ }^{197}$ VKQSGGGCC

... ${ }^{184}$ KRKRKKCLLL

$\ldots{ }^{185}$ GCINCCLIL 